\title{
Non-urgent pediatric emergency department utilization - A case review
}

\author{
Danette Dutra ${ }^{1}$, Ruth K. Rosenblum ${ }^{2}$ \\ 1. Department of Nursing, Fresno campus, California State University, Fresno, CA, U.S.A. 2. The Valley Foundation School \\ of Nursing at San Jose State University, One Washington Square, San Jose, CA, U.S.A.
}

Correspondence: Danette Dutra. Address: Department of Nursing, Fresno campus, California State University, Fresno, CA, U.S.A. Email: ddutra@csufresno.edu

Received: December 30, 2013

Accepted: May 7, 2014

Online Published: May 19, 2014

DOI : $10.5430 /$ cns.v2n3p45

URL: http://dx.doi.org/10.5430/cns.v2n3p45

\begin{abstract}
Parents who repetitively use the emergency department (ED) for non-urgent care create potential risk for their children. This phenomenon is influenced by inconvenient primary care office hours, insurance issues, and guaranteed same day services in the ED. Essentially, parents are using the ED as a primary care office, rather than a place for the care of urgent illness. This is a serious health concern as children do not receive continuity of care nor do they receive a focus on preventative healthcare when their primary place of obtaining care is solely in the ED. This case study presents an example of inappropriate, repeated ED use and offers potential solutions that enhance parental, ED provider, and primary care provider (PCP) accountability; enhancing the ability to provide appropriate care to a vulnerable population.
\end{abstract}

\section{Key words}

Emergency Department, Non-urgent, Pediatric primary care, Quality care health outcomes

\section{I ntroduction}

Pediatric patients are a vulnerable population in obvious need of continuity of care for growth and development, anticipatory guidance, and nutrition. Pediatric non-urgent emergency department visits are increasing, which places a child at greater risk for seamless healthcare ${ }^{[1]}$. Responsibility for solving this issue falls with both ED and PCPs. ED providers need to consistently refer the pediatric patient back to the PCP and investigate methods that would facilitate the required decrease in non-urgent ED visits. This will require the ED provider to be pro-active and resolute in the management of the pediatric patient with frequent ED visits. Most adult ED providers are uninformed of the extent of inappropriate ED use by the pediatric population, so merely contribute to the misuse by acquiescing to rapid discharge of the non-urgent pediatric patient. In a study by Kubicek et al., it was articulated that parents and primary care providers serve as "gatekeepers" to medical care for children ${ }^{[2]}$. Therefore, interventions designed for these primary caretakers' must consider the distinctiveness of all involved. Thru the understanding all sides, effective programs can be developed, and further evidence can be researched with the ultimate goal of improved patient outcomes.

A systematic literature review of ED visits for non-urgent conditions in the general population found it difficult to determine the exact driving factor for reasons why parents sought care in an ED for their children. Contributing factors may include younger age, convenience of the ED, referral by a PCP or other health care provider, and negative perceptions 
of non-ED sites. Cultural norms and satisfaction of care were also identified ${ }^{[3]}$. A recent position statement, by the Emergency Nurses Association (ENA), stated that according to a 2011 survey by the Centers for Disease Control and Prevention (CDC) at least 50 percent of the stated reasons why patients came to the ED for non-urgent issues were associated with the fact they did not know where else to go ${ }^{[4]}$.

\section{Purpose statement}

The purpose of this case study is to illustrate and offer problem solving techniques for both the primary care and ED providers who care for children and families. To begin, a case presentation is provided involving a 15 month old child that presents to the emergency department (ED); subsequently the case unfolds to offer insight into the misuse of the ED for healthcare and how the pediatric population is at increased risk for complications. Both the ED and the primary care provider (PCP) perspectives are discussed and possible solutions are offered.

Primary care providers bear a responsibility to be more available and practical in preventive education and anticipatory guidance provided to parents. PCP offices need to offer flexible schedules and be innovative in their approach to communication with anxious parents. Both the ED and the PCP provider should follow the advice, offered by Kathleen Sebelius the Secretary of Health and Human Services, to maintain transparency within communication between providers via the electronic medical record systems ${ }^{[5]}$. Ultimately the over-all goal, of this article, is to provide insight into an at-risk healthcare population, and offer concrete options of improved care. Initiating some of the suggestions within this case study could possibly lead to a decreased amount of misuse by parents of the ED.

\section{Case presentation}

Presented is a case study that seeks to offer an example of a commonly occurring healthcare problem; parental overuse of the ED for non-urgent healthcare issues within the pediatric population. Subsequently in this article possible solutions will be established.

\subsection{I nitial contact with child}

A 15 month old hispanic female presents to the ED with a chief complaint of cough, congestion, and runny nose for 2 days. The triage nurse obtains the following vital signs: Temperature 99.4 degrees Fahrenheit-rectally, pulse 92, respiratory rate 26, and a blood pressure of 102/65. The child weighs 9.5 kilograms. The triage nurse documents that the child is alert, playful, and is in no acute distress. She has no significant past medical history except frequent "colds". The child is assigned the triage category of non-urgent.

A nurse practitioner (NP) evaluates the child and a viral upper respiratory infection is diagnosed with the recommendation of symptomatic care. During the discharge process the mother complains to the discharge registered nurse (RN) that she doesn't understand why an antibiotic was not prescribed. The RN explains the difference between viral and bacterial infections but the mother is increasingly dissatisfied and so the NP intervenes. Mom informs the NP that she brings the child to the ED for her care because they can see her right away and "they give medications to her child". The mother's statement motivates the NP to review the child's ED visit records. She finds that this toddler has had 13 ED visits in her 15 months of life. Each of the 13 visits was classified as non-urgent acuity level. At nine of the visits an antibiotic was prescribed, including at least once by the same NP identified in this case study. Upon further review of the visits, a consistent theme of cough, nasal congestion, fever, and an occasional rash were primary chief complaints. Documentation showed that at several visits the child was noted to be under weight and consistently behind in immunization status. No mention of developmental milestones was evident in any of the 13 visits. After reviewing the child's ED records the NP investigated in greater detail the general heath of the child. 


\subsection{Family history}

Mother states that she and the patient's father are healthy, and that her mother and mother-in-law have diabetes. The toddler's maternal grandfather has hypertension and a history of heart attack.

Personal/Social/Developmental History Mother and father are hispanic, mom, age 26 was born in California; dad, age 27 was born in Mexico and only recently came to this area. He is here on a work visa and is employed as a farm laborer. This is their first child. The pregnancy was uneventful and delivery was without complications. Toddler's birth weight was 3.13 $\mathrm{kg}$, 29th percentile. She was assigned a PCP at the local rural health clinic. Mom does not like going to the clinic because of long waits for appointments and was under the impression that the clinic was only for "baby shots" and not acute illness. Additionally, transportation to clinic is an issue so she prefers using the ED because she can "come anytime".

\subsection{Pertinent review of systems}

Chief complaint on this ED visit is cough, nasal congestion, and runny nose for 2 days. Mother states it is a dry cough that is no worse at night and does not limit activity level. According to mother the child has a diminished appetite but no vomiting or diarrhea. She is noted to be consuming and tolerating fluids during the examination, specifically a baby bottle filled with red fruit punch. She has no known allergies and does not take any medication on regular basis. According to mom the toddler is behind in immunizations.

\subsection{Pertinent physical examination findings}

The toddler's examination was consistent with upper respiratory infection. The NP discusses symptomatic relief and provides age appropriate after-care instructions for viral upper respiratory infection. She also advises the mother to follow-up with the toddler's primary health care provider. She reviews with mom that the child is overdue for immunizations and strongly recommends regular visits to the primary care provider. The entire encounter with the child and her mother was less than 15 minutes. It took place within the triage area of a busy ED that is designated for rapid medical evaluation (RME).

\section{Discussion}

This case highlights concerns regarding use of the ED by parents seeking care for a child's non-urgent condition. This toddler's 13 visits to an ED never included education regarding preventive health care, nor developmental surveillance or assessment. The frequency of antibiotic prescriptions (nine antibiotic courses by age 15 months) would have been noted by the PCP if the primary care office had been used appropriately. Lack of continuity of care for this toddler may have endangered her wellbeing as well as increased antibiotic resistance. Pediatric patients who have greater than three ED visits per year for non-urgent health issues are at greater risk of not following up with the PCP and are more likely to be continued repeat ED users for non-urgent health care ${ }^{[6]}$. This case study exemplifies the stalemate. Immediately evident challenges in this case study include: deficient immunization status, weight below the 19th percentile and a bottle with fruit punch; suggesting the need for possible nutritional education. A need for a comprehensive assessment and consistent education for nutrition, anemia, dental health, developmental milestones, immunization, and safety is clearly evident. Secondary challenges are the ramifications associated with the overcrowding these non-urgent visits cause in the ED.

Emergency department visits have increased from 44 million patients in 1968 to approximately 134 million visits currently. Of those current visits, $27 \%$ are estimated to be deemed non-urgent. This equates to over 4 billion in extra healthcare costs ${ }^{[3]}$. The pediatric population visits comprise approximately one quarter of all ED visits. Of those ED visits up to $82 \%$ are considered non-urgent and could be handled in a primary care office ${ }^{[7]}$.

Once an individual starts the trajectory of inappropriate use of the ED, it will continue unless intervention occurs ${ }^{[3]}$. In a qualitative study of 31 families, Berry et al. asked why children were brought to the ED rather than the PCP. The client 
responses were typically critical of healthcare received in PCP offices. However, this investigation did not emphasize the need for ED providers to assist in problem solving to help parents understand continuity of care and health maintenance with the PCP.

Ross et al. ${ }^{[6]}$ investigated whether a standard intervention of education, counseling, and service coordination by a social worker in the ED would impact future ED visits for at-risk pediatric patients. The sample included 104 at-risk pediatric patients who had greater than three ED visits in one year for non-urgent conditions. Of these, 52 were assigned to the control group and 52 to the treatment group. Following the intervention of education, counseling, and coordination of health care services by the social worker during their ED visit, the treatment group decreased their use of the ED for non-urgent visits by $66 \%$. This was a statistically significant reduction. The list below provides a summary of common elements related to well childcare that are overlooked due to frequent ED as compared to PCP visits.

Elements of well child care overlooked due to frequent ED vs. PCP visits:

- Immunizations

- $\quad$ Acute illness education, prevention, and follow up

- Serial developmental assessments

- Diet/nutrition/obesity education and monitoring

- Anticipatory guidance for acute illnesses (URIs, rash, etc.)

- Anticipatory guidance for stages of growth and development along the age spectrum

- Early identification and treatment for chronic diseases that may start insidiously i.e. multiple ED visits for reactive airway disease, but no continuity of care or long term monitoring of asthma.

\subsection{Problem solving suggestions}

The inappropriate use of the ED by parents for a child's non-urgent conditions needs to be addressed by both the primary care and ED providers. This case discussion will focus first on the ED provider and secondly on the primary care office provider.

\subsection{Emergency department provider}

Often the ED provider categorizes pediatric patients into two unofficial categories. The first: 1) They are too sick to go home - complete the assessment and admit/transfer as soon as possible; 2) They are well enough to go home - arrange ED discharge as soon as possible. The underlying theme is as soon as possible. The ED provider is primarily concerned with the chief complaint of this visit rather than continuity of care, weight gain, inappropriate diet and nutrition, or lack of appropriate immunizations. The ED providers will not alter the immunization discrepancy or take the time to offer substantive, useful nutritional advice. Remember, the goal is to discharge the child as soon as possible ${ }^{[7,8]}$.

Ross et al. ${ }^{[6]}$ found that social worker involvement was effective in decreasing the misuse of the ED for non-urgent conditions. Interventions by social workers included education, counseling, and assistance with navigating the health care system. These tools fueled the effect of decreasing inappropriate ED use. However, smaller EDs do not have social work services so are unable to reap these benefits. Easily accessible and culturally relevant educational material discussing the importance of follow-up with a PCP and not the ED would be supportive. The preprinted accessible material would give providers in the ED an efficient method to educate parents of the importance of further follow-up with their PCP. Another approach is for the ED provider to take a thorough history with specific question related to access to health care, so that high-risk children like this 15 month old can be identified quickly. 
With the increasingly more common mainstay of electronic medical records (EMRs), the United States government has capitalized on the stance that a key component of the affordable healthcare act is transparency with regard to communication between providers. Kathleen Sebelius, Secretary of Health and Human Services, shares that with increased technology the confidential sharing of heath records may reduce the common errors associate with lack of communication among healthcare providers ${ }^{[5]}$. Even if it is not currently feasible to communicate via EMR to PCP, it is possible for the ED's electronic record keeping system to provide red flags or denote in some manner potential misuse of the ED for non-urgent visits. Thus, alerting the ED provider to the need of further education for the vulnerable pediatric patient. If all ED providers are committed to these interventions, perhaps a real change can occur, thus moving non-urgent health care needs back to the PCP and keeping the ED available for urgent matters. A summary of the potential improvements that could be initiated in the ED are listed below.

Summary of recommendations for ED providers to decrease inappropriate use of ED:

- $\quad$ Easily accessible educational material emphasizing the importance of follow-up with PCP.

- Thorough history of the pediatric patient to identify potential overuse of ED

- As a norm, when discussing plan of care with parents before discharge, stress importance of follow-up with PCP; be clear why it is important to see PCP on a regular basis.

- Assist in the development and implementation of a "red flag" computer generated alert that would identify frequent visits of the pediatric population.

- Develop social services contacts, if not available afterhours, a referral process exists.

- Program ED’s EMR to alert frequent non-urgent visits of the pediatric population, allowing necessary education to parents regarding risks of using ED as primary care.

- Customer satisfaction that are either mailed or phone contact made following "red flag” pediatric patients.

\subsection{Primary care provider}

A common reason cited for an ED rather than PCP visit was lack of same-day appointments. Parents of ill children are often told it will be several days to a week for the next available appointment. A 2009 study, executed across the US, indicated the average wait time to see a family nurse practitioner was 20.3 days ${ }^{[9]}$. PCP surrogates (medical assistants, front desk staff) then advise the parent go to the ED for care. If the parent's call is made after 1400, the likelihood of advising to go to an ED was even higher ${ }^{[10]}$. Additionally, parents perceive inconvenience relating to PCP hours of availability, location, and needed transportation ${ }^{[2]}$. Another reason that parents go to the ED for care is that they are unable to determine whether the condition was emergent or not. Parental lack of understanding regarding urgent vs. non-urgent treatment, was cited as factors in frequent ED use ${ }^{[1,7]}$.

Several options have been suggested to ameliorate these issues and deflect care back to the PCP. Education at each PCP and ED visit is essential. While parents might not remember specifics, easily accessible and readable materials in multiple languages may eliminate some unnecessary ED visits. One parent related that if she could have just talked to someone knowledgeable from her primary provider's office, she might not have rushed to the ED ${ }^{[7]}$. An after-hours automated phone tree of most common symptom relief measure would be useful. The automated pre-recorded messages would have to be high-quality and clear for the parents to follow, and recorded in multiple languages for a culturally diverse population. Several large HMOs have successfully done this for many years for both the adult and pediatric patient population. An on-call NP for immediate questions (both during office hours and after office hours) is another solution to decrease frequent unnecessary ED visits ${ }^{[8]}$. Published “call-in” hours to speak with an RN or NP also may enhance parental understanding of how and when to get immediate answers to questions by the appropriate caregiver. While the 
burden of funding these initiatives falls to the individual PCP office or group, anticipated savings in health care dollars would be abundant. Fixing the "who pays" discrepancy is a challenge ${ }^{[8,10,11]}$.

In the PCP office, the EMR could also be a relevant addition to stimulate increased awareness of assessment and teaching needs about ED visits. The EMR system could also be used to support electronic mailers that focus on common childhood aliments. Perhaps even an electronic email supported communication system with an on-call nurse practitioner, both during office hours and for potential after-hours electronic conversations ${ }^{[10]}$.

Long wait times for an appointment and then additional waiting at the PCP office have also been identified as key factors. The ED is perceived as offering quicker, faster, more efficient, and better equipped to provide immediate care. Tests and procedures are done "right here, right now" rather than going to multiple other locations for labs and radiologic tests ${ }^{[8]}$. Open-access scheduling is described as another potential solution for long wait times in the PCP office. This is an alternative scheduling system that offers patients' same-day appointments with availability adjusted according to an algorithm of expected need (e.g. Monday mornings or late afternoon time slots), provider availability, and season. Use of computers or "apps" could be implemented to regulate the flow of patients at the PCP office. Extended or flexible walk-in hours and/or weekend and evening hours are other interventions to consider ${ }^{[7]}$.

Recommendations offered by Sturm et al included optimizing the ratio of "sick" slots to "well" slots based on season or other factors, making same day turnaround for labs available, and endorsing policies to accept walk-in sick visits ${ }^{\text {[12] }}$. These ideas potentially can produce societal and individual cost savings and help patients and families sustain continuity of care through the PCP office. In larger metropolitan areas, many private pediatric practices have pooled efforts to provide weekend, evening, and holiday care jointly, sharing the burden yet responding to patient and family needs, and making ED visits for non-urgent conditions less likely. Berry et al. cites negative PCP staff attitudes and communication issues as detractors from using PCP rather than ED services ${ }^{[7]}$.

A demonstration that the lack of insight into the issue of ED overuse, by the pediatric population, is still very much present in the United States today consider a 2010 study by Broussard ${ }^{[13]}$. According to Broussard they found that neither parents nor primary physicians found non-urgent ED visits' as a significant enough problem to warrant further study or a change in practice. The physicians in this study approved of parents' use of the ED, and more importantly felt that the use of the ED was not a breach in continuity of primary care for the children. This is a disheartening finding. Listed below is a summary of potential improvements to PCP office to help address key issues. Summary of recommendations for PCP office to increase access:

- Extended and/or flexible hours with multilevel provider staffing

o Open-access scheduling via phone or "app"

- “Call-in" hours for questions and concerns re: common childhood illnesses not immediately requiring a visit.

o Direct provider phone contact for immediate response

o Review of treatment for minor illnesses via phone, email, or smartphone (text)

- Improved communication between PCP clinic and patients/families

o General customer service training

o Patient and family centered care

o Clear instructions on when and how to reach personnel, especially after hours

o "Just in time” contact for provider availability via text message

o Continuity of care with same provider at each visit when possible 


\section{Conclusion}

Ultimately, the data presented in the literature indicates that parental use of the ED for non-urgent medical conditions in their children is a complex issue that lacks focus and consensus. Common knowledge dictates that ED care is costlier than care by a PCP and is less effective in providing appropriate primary and chronic condition care for children. A key set of principles guiding this discussion relates to child health education provided to parents, convenience to reach care when needed, and trust and confidence in medical providers ${ }^{[2]}$. ED providers can assist in directing these patients to primary care by 'flagging' the chart in the EMR and ensuring that proper referrals are made to a PCP. Ensuring that the patient and family attend the given appointment is much more difficult until and unless infrastructure changes occur; such as shared data bases via the EMR. Other possible infrastructural changes that could occur include evening or weekend hours at the PCP's office, assistance with transportation, and the potential for "walk-in" appointments when needed during regular office hours and extended hours. This presents a financial and organizational challenge to the PCP office that must have a congruent organizational commitment. Enhanced awareness of the potential concerns regarding the lack of continuity of care regarding common childhood illnesses, developmental milestones, nutrition, anticipatory guidance and preventative healthcare issues is essential in stemming the flow of pediatric patients and families opting to use the ED as their most-utilized healthcare environment. The case study described in this article illustrates the issues and offers problem solving techniques for primary care and ED providers who care for children and families. Frequent antibiotic prescriptions, delayed immunizations, and inappropriate feeding practices must be considered unacceptable today. We can do better for children and families in our care.

\section{References}

[1] Brim C. A descriptive analysis of the non-urgent use of emergency departments. Nurse Researcher. 2008; 15(3): 72-88. PMid:18459488 http://dx.doi.org/10.7748/nr2008.04.15.3.72.c6458

[2] Kubicek K, Liu D, Supan J, Weiss G, Lu Y, Kipke MD. (2012). A profile of non-urgent emergency department usage in an urban pediatric hospital. Pediatric Emergency Care. 2012; 28: 977-984. PMid:23023463

http://dx.doi.org/10.1097/PEC.0b013e31826c9aab

[3] Uscher-Pines L, Pines J, Kellermann A, Gillen E, Mehrotra A. Emergency department visits for nonurgent conditions: Systematic literature review. The American Journal of Managed Care. 2013; 19: 47-59. PMid:23379744

[4] McMillin T. What's the right solution for ER overuse? ENA NewsLine[Internet]. 2014[cited 2014 Mar 25]. Availble from: www.multibriefs.com/briefs/ENA

[5] Sebelius K. Good news on innovation and health care. United States Health and Human Services[Internet]. 2013. Available from: www.HHS.gov/healthcare

[6] Ross JW, Roberts D, Campbell J, Sofranko-Solomon K, Brouhard BH. Effects of social work intervention on nonemergent pediatric emergency department utilization. Health \& Social Work. 2014; 29(4): 263-273. http://dx.doi.org/10.1093/hsw/29.4.263

[7] Berry A, Brousseau D, Brotanek JM, Tomany-Korman S, Flores G. Why do parents bring children to the emergency department for nonurgent conditions? A qualitative study. Ambulatory Pediatrics. 2008; 8(6): 360-367. PMid:19084785 http://dx.doi.org/10.1016/j.ambp.2008.07.001

[8] Philipes H, Remmen R, De Paepe P, Buylaert W, Van Royen P. Out of hours care: A profile analysis of patients attending the emergency department and the general practitioner on call. BioMed Central Family Practice. 2010; 11(88): 1-8. Avaible from: www.biomedcentral.com 1471-2296/11/88

[9] Merritt Hawkins and Associates, 2009 Survey of Physician Appointment Wait Times. Irving, TX: Merrit Hawkins and Associates; 2009.

[10] O’Mallery AS. After-hours access to primary care practices linked with lower emergency department use and less unmet medical need. Health Affairs[Internet]. 2013; 32(1): 1-7. Avai lable from: www.content.healthaffairs.org

[11] Pines JM, Budord K. Predictors of frequent emergency department utilization in southeastern Pennsylvania. Journal of Asthma. 2006; 43: 219-223. PMid:16754525 http://dx.doi.org/10.1080/02770900600567015

[12] Sturm JJ, Hirsh DA, Lee EK, Massey R, Weselman B, Simon HK. Practice characteristics that influence nonurgent pediatric department utilization. Academic Pediatrics, 2010; 10: 70-74. PMid:20129483 http://dx.doi.org/10.1016/j.acap.2009.10.001

[13] Brosseau DC, Nummer MR, Yunk NL, Nattinger AB, Greer A. Non-urgent emergency department care: Analysis of parent and primary physician perspectives. Pediatrics. 2011; 127: e374-e380. 